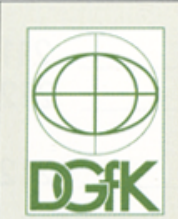

KN Kartographische Nachrichten Journal of Cartography and Geographic Information 66. Jahrgang, August 2016, Heft 4 Herausgeber: Deutsche Gesellschaft für Kartographie e.V. (DGfK) - Gesellschaft für Kartographie und Geomatik, vertreten furch den Präsidenten durch den Präsidenten
Prof. Dr. Manfred Weisensee

Organ der Deutschen Gesellschaft für Kartographic e.V. (DGfK), der Schweizerischen Gesellschaft für Kartografie (SGK) und der Osterreichischen Kartographischen Kommission (OKK) in der

Osterreichischen Gesellschaft für Geographie (0GG).

Schriftleitung

Hauptschriftleiter

Prof. Dr. rer. nat. habil. Mark Vetter (zustăndig für Aufsătze, Berichtel. Hochschule Karlsruhe - Technik und Wirtschaft,

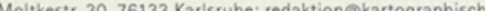
nachrichten.de, Fon: 0049(0) 7219252599

Weitere Schriftleiter

Prof. Dr. phil. Frank Dickmann (Zweiter Schriftleiter, zustăndig für Nachrichten aus Hochschulen und Institutionen, Rezensionen).

Ruhr-Universităt Bochum, Geographisches Institut,

Postfach, 44780 Bochum; frank. dickmann@iub de

Fon: 0049(0) 234 32-23379; Fax: 0049(0) $23432-14964$

Dipl.-Ing. Andreas Gollenstede (zuständig für Geoinformation

aktuell) Haareneschstr. 91, 26121 Oldenburg; kn@gollenstede.com Fon: 0049(0) 4417779-545; Fax: 0049(0) 441 7779-750

Dr.-Ing. Thomas Chudy (zuständig für DGfK-, SKG- und OGG-

Nachrichten). Martin-Luther-Universität Halle-Wittenberg, Institut

für Agrar- und Ernăhrungswissenschaften, Karl-Freiherr-von-

Fritsch-StraBe 4, 06120 Halle/Saale, Fon: $0049(0) 3455522448$ :

Fritsch-Straße 4, $06120 \mathrm{Ha}$

Prof. Dr. Reinhard Zölitz (zuständig für Nachrichten aus Hoch-

schulen und Institutionen), Institut für Geographie und Geologie,

Kartographie und GIS, Friedrich-Ludwig-Jahn-Str. 16

D-17489 Greifswald. Fon 03834864523 , Fax: 03834864501

E-Mail: zoelitz@uni-greifswald.de

Mitarbeit für die Schweiz: Prof. Dr. Lorenz Hurni. Institut für Kartografie, ETH Hönggerberg, 8093 Zürich; hurni@karto.baug.ethz ch. Fon: 0041 16333033; Fax: 00411631153

Mitarbeit für Österreich: Univ.-Prof. Dr. Wolfgang Kainz

Institut für Geographie und Regionalforschung. Universităt Wien.

Universitätsstraße 7/5, 1010 Wien; wolfgang.kainz@ @univie.ac.at

Fon: 0043 14277-8640; Fax: 0043 14277-9531

Ehrenschriftleiter

Prof. i. R. Dr. Jürgen Dodt, Witten

Dr, ree, nat, h. c. Rolf Harbeck

Editorial Boord

Prof. Dr. H. Asche, Potsdam; Prof. Dr. Manfred Buchroithner,

Dresden; Prof. Dr. Dirk Burghardt, Dresden; Prof. i. R. Dr. Jürgen

Dodt, Witten; Prof. Dr. D. Dransch, Potsdam; Prof. Dr. M. Ehlers,

Osnabrück, Berlin; Prof. Dr. S. L. Fabrikant, Zürich, Schweiz;

Ass-Prof. Dr. S. Fuhrmann, Fairfax, USA; Prof. Dr. G. Gartner, Wien,

Österreich; Prof. Dr. D. Grünreich, Ronnenberg; Dr. Rolf Harbeck

Bonn: Prof. Dr. A. Hüttermann Ludwigsburg: Prof. Dr. L Hurni

Bonn, Prof. Dr. A. Hüttermann, Ludwigsourg, Prof. Dr. L. Hurni,

Zurich; Prof. D. W. Kainz, Wien; Prof. Dr.P. Kammerer, München:

Prof. Dr. W. G. Koch, Dresden; Prof. Dr. Menno-Jan Kroak, Twente,
Netherlands; Prof. Dr. Jukka Krisp, Augsburg; Prof. Dr.-Ing.

Netherlands; Prof. Dr. Jukka Krisp, Augsburg: Prof. Dr.-Ing.

J. Schoppmeyer, Bonn; Prof. Dr. J. Schweikart, Berlin; Prof.

Dr.-Ing. M. Sester, Hannover; Prof. Dr. J. Siemer, Regina, Kanada;

Prof. Dr. R. Zolitz, Greifswald.

Manuskripte richten Sie bitte an den zustăndigen Schriftleiter.

Hinweise zur Gestaltung des Manuskripts finden Sie unter

wwwkartographische-nachrichten.de > Autorenhinweise.

Reviewverfahren

Wissenschaftliche Beitrăge werden im Rahmen eines Peer-ReviewVerfahrens begutachtet. Trotz sorgfältiger Redigierung übernehmen Herausgeber. Redaktion und Verlag keine Haftung für die inhaltliche Richtigkeit der Veroffentlichungen.

Die KN Kartographische Nachrichten ist in der internationalen Zitationsdatenbank Scopus gelistet.

Die DGfK ist Mitglied der

Internationalen Kartographischen

Vereinigung IKV/ICA.

Verlag: Kirschbaum Verlag GmbH, Siegfriedstraße 28,

D-53179 Bonn / Postfach 210209 , D-53157 Bonn.

fon: (0228) 95453-0, Fax: (0228) 95453-27:

Internet: $h$ ttp: $/ /$ www.kirschbaumde, E-Mail: infookirschbaum.de

Anzeigenleiter: Volker Rutkowski; zurzeit ist Anzeigenpreisliste

Nr. 51 vom 1.10.2015 gültig

Bezugspreise und ISSN: Inland/Ausland tahresabonnement

inkl. E-Paper und elektronischem Archiv 74,- Euro zzgl.

Versand 9,80,- Euro (jeweils einschl. MwSt).

Einzelheft 18,- Euro zzgl. Versand

Die Zeitschrift erscheint alle zwei Monate. ISSN 0022-9164.

Kündigungsfrist 6 Wochen zum Auslaufen des Abonnement-

Zeitraumes.

Satz: EMS Eckert Medienservice, 53359 Rheinbach

Druck: johnen-druck GmbH \& CO. KG, Industriegebiet Bornwiese.

54470 Bernkastel-Kues

\title{
Der kartographische Nachwuchs
}

\section{Liebe Leserin, lieber Leser,}

Am Ende des Praxissemesters, nach dem 5. Semester, stellen unsere Bachelorstudierenden des Geoinformationsmanagements ihre Erfahrungen aus dem Praxissemester in einem Vortrag dar. Dieses Element im Studium ist sehr wichtig für die Verbindung zwischen Wirtschaft, Verwaltung und Hochschulen. Eine derartige Pflichtlehrveranstaltung ist für die hochschulverantwortlich Dozierenden in unserem Fach ein gutes Barometer für die gegenwärtigen Trends und Entwicklungen in der beruflichen Wirklichkeit. Es lassen sich so Erkenntnisse über ein etwaiges Nachjustieren der curricularen Inhalte oder Schwerpunkte des Studienprogramms gewinnen.

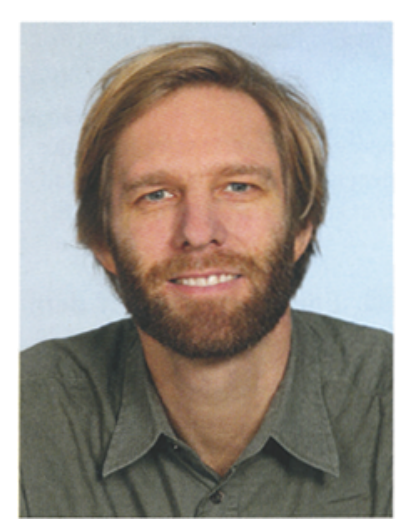

Mark Vetter
Die gute Nachricht ist, dass die Grundstimmung der jungen Studentinnen und Studenten nach der Erfahrung im beruflichen Alltag durchweg positiv ist. Dies gilt sowohl in der Eigen- als in der Fremdwahrnehmung. Große, existenzielle Sorgen ob der eigenen beruflichen Zukunft habe ich sehr selten erlebt. Ich habe nie gehört, dass die Tätigkeit während des Praxissemesters langweilig oder wenig gewinnbringend gewesen wäre. Alle Studierenden fühlten sich in ihrer Wahl des richtigen Studienfachs bestätigt.

Warum ist diese Lehrveranstaltung für alle Beteiligten so wichtig? Die Unternehmen sind froh, dass die Studierenden im Betrieb wertschöpfend eingesetzt werden können. Die Studierenden freuen sich, Erfahrungen aus dem Arbeitsalltag zu sammeln. Wir Hochschullehrende freuen uns, die Rückmeldung von Unternehmen und Ämtern zu bekommen, dass die Studieninhalte größtenteils für die aktuellen Aufgaben relevant sind.

Allerdings wird auch diese Rückkoppelung dafür verwendet, unsere Studieninhalte immer wieder anzupassen. Die Unternehmen und die Behörden profitieren insbesondere davon, dass die Studierenden in unserem Fach an allen Hochschulen über neueste IT-Methoden im Umgang mit Geodaten unterrichtet werden. Die Bedeutung in unserer digitalen Gesellschaft mit neuester Soft- und Hardware umgehen zu können nimmt stetig zu. Ebenso an Bedeutung gewinnt der professionelle Umgang mit modernen Programmiersprachen und speziell für die Geobranche entwickelten Programmen und Standards. So nehmen diese Anteile im Studium ständig mehr und mehr Raum ein. Und auch in den nächsten Jahren werden die Hochschullehrenden sehr aufmerksam die Entwicklungen in der Geoinformationstechnologie beobachten, um das Studium weiterhin den Erfordernissen für unseren Nachwuchs anzupassen.

In der vierten Ausgabe der Kartographischen Nachrichten 2016 erwartet Sie Folgendes: Der Aufsatz von Fabian Jetter und Stepan Bosch zeigt auf, wie das Solarpotenzial auf den Dächern mit einem GIS über gebäude- und siedlungsbezogene Daten analysiert werden kann. Philipp Seyerlein stellt Möglichkeiten und Grenzen des neuen OGC-Standards Geopackage vor. Der Beitrag von Lowie Brink untersucht die historische Entwicklung bei der Generalisierung in niederländischen Schulwandkarten und Marcel Chaouali hat sich mit der Visualisierung von Geodaten der niedersächsischen Landeshauptstadt Hannover beschäftigt.

Ich wünsche Ihnen viel Freude bei der Lektüre dieses Hefts,

herzlichst,

Ihr

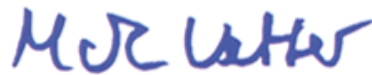

